International Journal of Advanced Trends in Computer Science and Engineering

Available Online at http://www.warse.org/IJATCSE/static/pdf/file/ijatcse38942020.pdf

https://doi.org/10.30534/ijatcse/2020/38942020

\title{
Use of Spline models in the Analysis of Signals collected during the measurement of Technological Processes
}

\author{
Utkir Khamdamov ${ }^{1}$, Avaz Mirzayev ${ }^{2}$, Sirojiddin Xalilov*3. \\ ${ }^{1}$ Department of Information Technologies, Tashkent University of Information Technology named after \\ Muhammad al-Khorezmi, Tashkent, Uzbekistan, khamdamovutkir@tuit.uz \\ ${ }^{2}$ Department of Information Technologies, Tashkent University of Information Technology named after \\ Muhammad al-Khorezmi, Tashkent, Uzbekistan, mirzoavazbek.@mail.ru \\ ${ }^{3}$ Department of Information Technologies, Tashkent University of Information Technology named after \\ Muhammad al-Khorezmi, Tashkent, Uzbekistan, kh.surajiddin@gmail.com
}

\begin{abstract}
This article is devoted to the use of spline models for signals measured by technological processes. The spline models embedded in the technological processes have high accuracy of signal interpolation, which allow professionals to make informed decisions as a result of digital signal processing. As an example, an interpolation spline model was built for digital processing signals from technological processes. A smoothing algorithm for solving linear equations for constructing an interpolation spline is presented.
\end{abstract}

Key words: Signal, seismic signal, bio signal, magnetic exploration, gravity exploration, spline function, interpolation spline, interpolation, technological process, classical polynomial, cubic spline, coefficients, processor, diagram.

\section{INTRODUCTION}

The introduction of information and communication technologies in the industry provides practical assistance in real-time decision-making, forecasting, long-term strategic planning, finding optimal solutions. In particular, in geophysics, seismology, medicine, mechanical engineering, the study of existing data, methods of signal processing, as well as the achievement of specific solutions are one of the most important issues facing experts in the field. The modern stage development of algorithms for the structure and operation machines, complexes and systems, which are part of test stands or located on moving objects, is characterized by rapid analysis of complex processes and areas and increasing requirements for real-time processing large amounts of information. The development of algorithmic, hardware and software tools and techniques remains relevant. Signal recovery and analysis, geophysical and seismic signal processing, the study of the results that stand experiments, the study images are the basis of digital processing processes. Generalized spectral methods and spline function methods are widely used in signal analysis and recovery[1][3][7].

Grinding filters are widely used in technological process in automation systems. Today there are many grinding algorithms are available, but the need to expand them remains high. This need suggest signals in real automated systems are often characterized by content instability and diversity, both in useful content and in error[1][2].

\section{PROPOSED METHOD}

The proposed grinding algorithms are based on spline theory. The number of works and articles on signal grinding is growing in our country and around the world. The following real-time cardiogram and reagram biosignal compression and grinding filters in which the grinding process is performed in two stages:

-Determine the location of the spline boundaries;

-Smoothing signals within the received limits.

It is based on interpretation of new and traditional technologies at the general structural level. Therefore, it is necessary to briefly consider the methodologies that reflect the processes of technology creation, which have a broad meaning as specific systems of a certain class as multifunctional production systems.

According to the systemic concept, technology is a system description and implementation of information and material resources in a systematic way, divided into simple processes with the separation of dual technological function and supporting subsystems. Clearly, this technology is described in Figure 1.

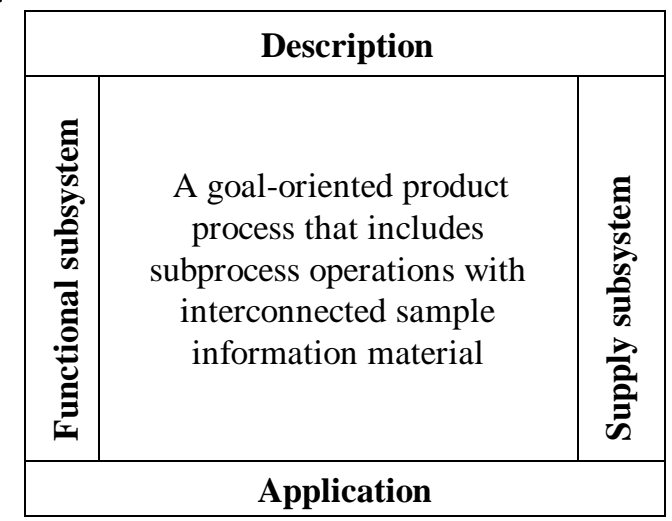

Figure 1:. A general understanding of technology 
According to the theory of processes of information and material types, the relationships between them, as well as the system of structural and mathematical descriptions of different external and internal conditions, technology is a practical science that corresponds to the system level of its presentation. Descriptive data with support for the production process and production visibility and integration should be based on the results of many systems performance, not just continuous techno parks, but the approach of constantly evolving systems[2][9] [10].

At the stage of determining location of the signal boundaries, it is necessary to collect the reporting blocks of non-interfering (noisy) signals. This means that grinding is performed with a delay equal to the time of collection of report blocks, which is allowed in biosignal processing, but in automated systems and technological processes, processing of operational information is not always allowed in the operation of contour filters.

In addition, the filter is designed to process smooth continuous signals in a difficult form that takes into account additive unrelated noise. Furthermore, the signal model is not fully compatible with measurement information signals in technological process automation systems, in particular the measurement information signals are not only smooth and continuous but also have interruptions and jumps and can be added to unrelated noise. The grinding splines proposed in the work are capable of continuous and intermittent operation, taking into account the characteristics of the measurement information signals[10][11][13]..

$$
\begin{gathered}
z\left(x_{i}\right)=z_{t}\left(x_{i}\right)+\varepsilon_{f}\left(x_{i}\right)+\varepsilon_{g}\left(x_{i}\right), \\
z_{t}\left(x_{i}\right)=\left\{z_{t}\left(x_{i}\right)=\left(\sum_{l=0}^{n(k)} a_{l}(k) * i^{l}\right)+\delta_{t}\left(x_{i}\right),|i=(\overline{1, l}), k=(\overline{1, k})|\right\} \\
\delta_{t}\left(x_{i}\right)= \begin{cases}\overline{\delta_{t}}\left(x_{i}\right), & i_{n, c} \leq i \leq i_{k, c} \\
0, & \text { if } i_{k, c} \leq i \leq i_{n, c}\end{cases}
\end{gathered}
$$

Required:

1. Formation of signal filtering and grinding algorithms based on compression grinding splines[7][9].

2. Select the spline filter adjustment method.

3. Comparison of existing filters based on modeling.

Figure 2 shows the structure of the Spline filter based on the Compression Grinding Splines

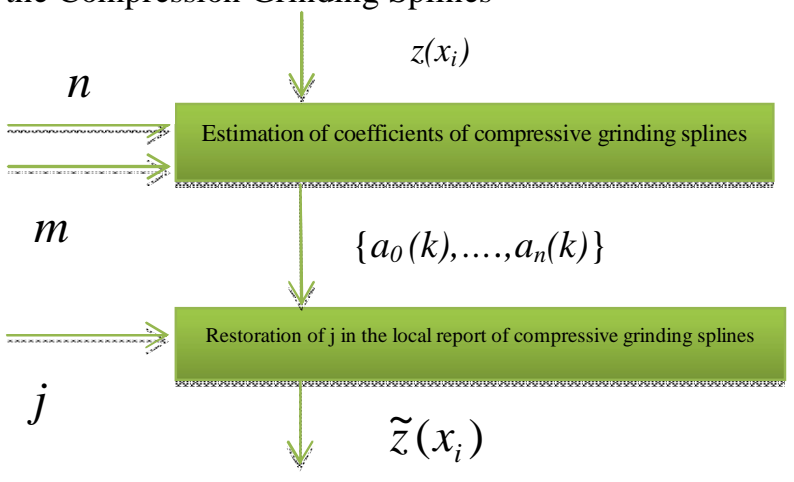

Figure 2: Spline filter structure based on compression grinding splines
The input data for the calculation of the coefficients to the compressive grinding splines are $\mathrm{m}$ in the last reports of the measurement information signals: $z\left(x_{i}\right), z\left(x_{i-1}\right), \ldots \ldots, z\left(x_{i-m}\right), z\left(x_{i-m+1}\right)$. The formulas for estimating the coefficients of $a_{i}(k)$ compressive grinding splines were obtained on the basis of KKU. We obtain the polished estimate based on the following formula $\tilde{z}\left(x_{i}\right)$ as a result of restoring $\mathrm{j}$ in the local report of the Compressed Grinding Splines:[12] [13]

$$
\tilde{z}\left(x_{i}\right)=\sum_{l=0}^{n} a_{i}(k) * j^{l}
$$

The delay sign is equal $\theta$ :

$$
\begin{gathered}
\theta=m-j-1 \\
j=m-1
\end{gathered}
$$

The algorithm works without delays.

Thus, the algorithm for working in a spline filter involves the process of calculating the coefficients of the compression grinding splines and finding the local $j$ at each time interval, if the local report $\mathrm{j}$ is located in the center of the compression grinding splines, $(j=m-1$ or $j=0)$ we can more accurately estimate [4] and accuracy of the assessment will be low if (or) within the limits.

It is recommended to use interference-protected compression grinding splines to increase the interference protection when the spline filter operates without delays. Figure 3 shows a spline filter structure based on interlocking compression grinding splines.

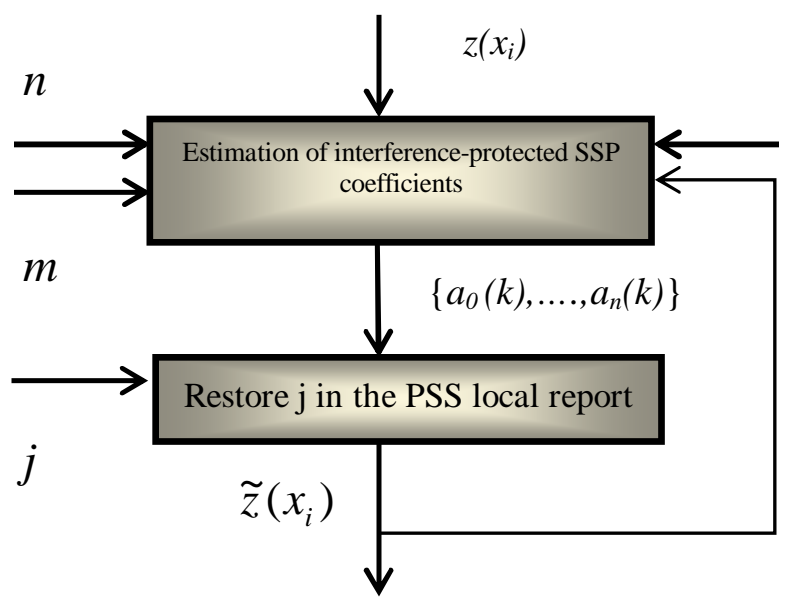

Figure 3: Spline filter structure based on interferenceprotected compressible grinding splines

Formulas for estimating the coefficients of interference protection of compressible grinding splines $a_{l}(k)$ where as an auxiliary report $z_{k}\left(x_{i+q}\right)$ used grinding estimates found in the previous steps

$$
z_{k}\left(x_{i+q}\right)=\tilde{z}\left(x_{i-1}\right)
$$


We obtain a polished estimate based on the following formula as a result of the local report recovery $(m-2)$ of the compressible grinding splines:[4] [5] [6]

$$
\tilde{z}\left(x_{i}\right)=\sum_{l=0}^{n} a_{i}(k) *(m-2)^{l}
$$

The value of the spline filter is based on simple compression grinding splines, the accuracy of which is less accurate in delay-free operation than in delays, i.e. the local calculation $\mathrm{j}$ in delay-free operation is located on the boundary of compression grinding splines. When compression grinding splines are protected from interference, the addition of an auxiliary report does not limit the local reporting, and often the result of modeling spline filter performance shows that a spline filter based on simple compression grinding splines allows for more accurate estimation. $z_{k}\left(x_{i+q}\right)=\tilde{z}\left(x_{i-1}\right)$

The useful composition of the model signals includes both "simple" signals (sinusoidal, linear, exponential, quasi-stationary, pulse, rectangular wave) and the interaction of functional interferences in different layers consisting of "simple" "difficult" $\varepsilon_{\phi}\left(x_{i}\right)$ and coarse waste $\varepsilon_{2}\left(x_{i}\right)$ reflects in niozida. If the spline filter thus created is used to filter and grind high-point measurement information signals, then the model signals can sufficiently amplify the exact characteristics of the measurement information signals as follows:

$-z_{t}\left(x_{i}\right)$ in the form of useful content (linear, exponential, cinusoidal, right-angled waves and their combinations);

- propagation and distribution of wave noise $\varepsilon_{\phi}\left(x_{i}\right)$ (normal distribution);

- The presence of the value and frequency of occurrence of coarse waste $\varepsilon_{2}\left(x_{i}\right)$.

To display the results of spline filter activity, the same signals were processed through filters of the first exponential grinding (ES1), second (ES2), third (ES3) order, and moderate deviation. The efficiency of the filter operation was evaluated by the OMX (average module error).

Signal processing is carried out in two training and control phases. During the training phase, the module is processed several times to determine the best of the signal filter settings. In the control phase, the model signal is processed with a filter set with the same useful content $z_{t}\left(x_{i}\right)$ and the same structure interfering content (only with other characters), set in the reading phase.

All that is needed to produce a finished product to carry out the production process and put it into practice is to implement the technology, which corresponds to the system level of the description as a complete set of sample or working presentation. The finished product includes a variety of automated and automated systems, human-machine subsystems, mechanisms, products, energy and information resources, services and more.

The structuring of any technology is accomplished through standard subprocessor operations that provide functional and subsystems. By process we mean the change of information, energy, objects in the system (object) over time. Depending on the purpose of the system and the product to be obtained, the processes are divided into a class of information materials, materials and information. Accordingly, the technologies that perform different processes are also classified. In modern conditions, the following development tasks are important:

1. Information such as receiving (measuring, collecting, extracting), encoding, transmitting, collecting, storing, searching, processing (filtering, compressing, predicting, modifying) and displaying data;

2. Data such as data reception (measurement, collection, output), coding, transmission, collection, storage, search, processing (filtering, compression, prediction, modification) and data display;

3. The materials of standard subprocessor operations include a wide range of mechanical, physical, chemical subprocess operations, along with new advances in materials science, synergetics, nanosystems.

Typical subprocessor operations serve as the main process by which information and materials processes, and technology in general, are formed. In the structure of any efficient process, the interdependence between typical subprocessor operations corresponds to the sequential, parallel, inverse-parallel (recalculation, processing), interdependence, transition, hierarchical, and combined connections of process connections.

Functional (by type of activity, purpose) subsystem serves as a governing component for all technologies. Each functional subsystem receives a functionally complete total sample subprocessor operations consisting of information and materials.

Supply subsystems, as the name implies, they mainly provide support for a set of technical, software and other types of functional subsystems.

The systematic approach focuses on the operation of objects of functional subsystems (primarily control objects) and activating functional subsystems (primarily functional subsystem managers). Functional subsystems can be defined common standard objects and types of activities, which implement and implement the following processes on the basis of standard subprocessor operations: measurement, modification, execution, management, (arrangement, task, editing), forecasting, planning, organization, goal and resource formation, design, construction, installation and commissioning, testing, experimentation, identification, modeling, database and knowledge formation, algorithmization, programming, training , stimulate, analyze, optimize.

Typical supply subsystems are suitable for the following types of supply: conceptual, methodological, algorithmic, mathematical, software, technical, information, metrological, personnel, organizational, financial, legal, material and energy, ergonomic, environmental

Subsystems that provide functional and functional support of technological structures through standard process operations should be produced taking into account the following:

Typical sub-process operations, which serve as the basis for a more complex process and operational structure, will have information about the unifying and objects in nature; 
Functional and support subsystems include interconnected sets of operations and processes, as well as entirely tangible objects;

Supply subsystems are divided into general (for all tasks) and private (for individual tasks);

In the construction of any technology, it makes sense to use a variety of private support systems, along with special functional subsystems that make up special functional support subsystems, rather than general supply subsystems (with basic hardware and software).

To the approach discussed above, M. V. In the development of Lyakhovtsa's natural-mathematical modeling technology and S.P. Clarity has been included in Ogneva's work on the development of reconstructive-predictive rule technologies.

Another approach to the systematization and classification of technology is presented in the work of I.V.Shelevitskogo. Figure 4 shows a DFD diagram of the IDEF3 standard of spline technology for processing signal of measurement information. Here in after, we define spline technology as the short term S-technology. In (UOB 1) the spline-function base is selected and synthesized.

Figure 5 (UOB 1) shows the disassembly. The first operation of S-technology is a detailed analysis of the processed signal (UOB 1.1), primarily an analysis of its frequency characteristics and technical capabilities used in information processing systems (UOB 1.2). The main purpose of this operation is to shape the quality and characteristics of information processing.
If the analysis of the signal structure is not sufficient, it is recommended to identify the fragmented structure of the data (UOB 1.3). Corresponding identification algorithms are detailed in the study. Based on the nature of the signals (logarithmic, exponential harmonic), a incremental function is selected to synthesize the base splines (UOB 1.4). (UOB 1.5) performs a baseline spline synthesis presented in analytical or digital form. The next operation will be to replace the base (UOB 2). The purpose of this operation is to obtain a spline at the Hermit or Lagrage bases. Then either an interpolation algorithm (UOB 4) or a (UOB 3) agortim is created, after which filtering, identification and interpolation processes are carried out depending on the importance of the problems to be solved.

The spline technology, developed by I. V. Shelevitskim, is designed to process invariant smooth signals in complex shapes, which can include flexible non-correlated noise. The technology solves the problems of compression and identification of signal parameters, filtering and synthesis of interpolation algorithms.

It is a bit difficult to implement the proposed system in automated systems of technological processes and production, as it does not involve real-time operation, as well as the basic properties of these systems (especially the presence of rough (excessive) noise, signal playback and interruption, rapid change of directions), and in such systems does not include the primary processing of soluble signals.

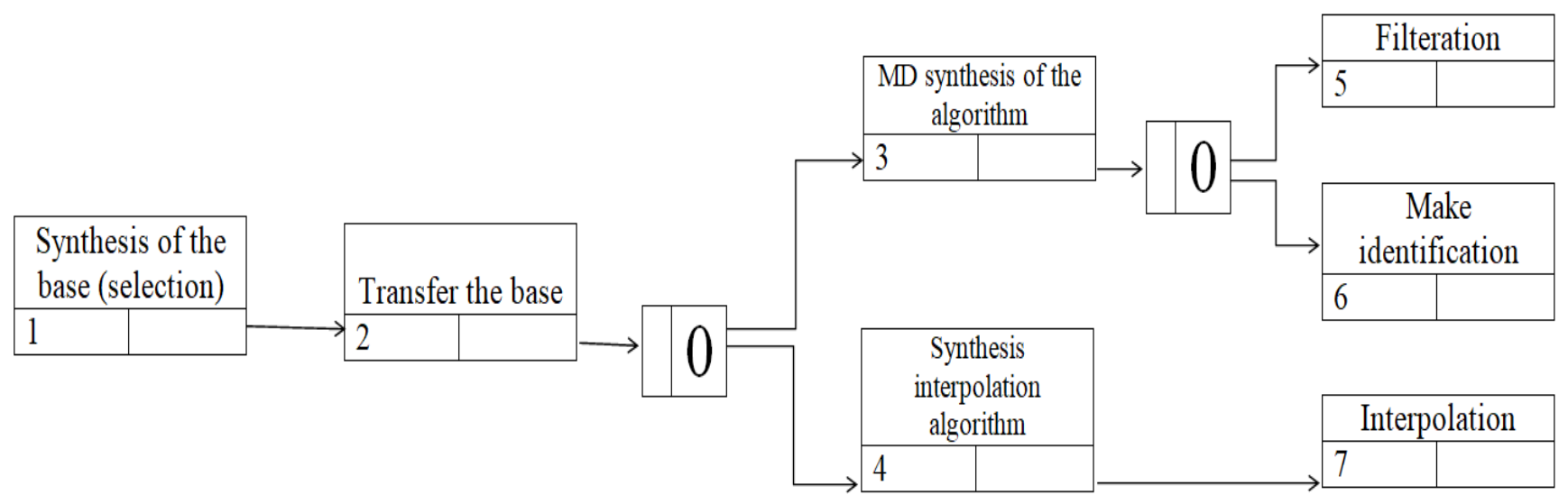

Figure 4: Information PFDD-diagram of spline technology
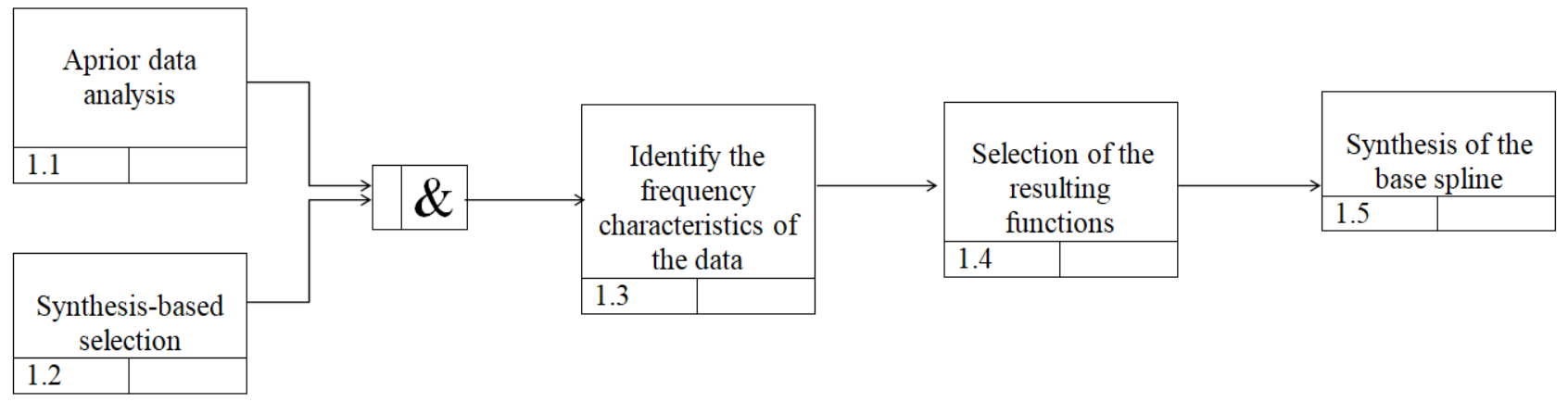

Figure 5: UOB Decomposition "Synthesis of base (selection) 
In accordance with the systems of spline theory and previous development experience and new training integrated concepts applied to the process automation system, spline technology has been developed, the general concept is shown in Figure 6, detailed information about the following subsystems:

An optimized subsystem based on continuous and search processes performs the identification of optimized solutions.
In the subsystem of the structural synthesis of the spline, a spline selection is performed with such classification marks (features) that allow the best solution of certain problems. In the system of synthesis of spline-approximation algorithms on the basis of $\mathrm{KKU}$ or $\mathrm{KMU}$, a corresponding algorithm is created, which allows to calculate the spline coefficients and the splay itself in the system content.

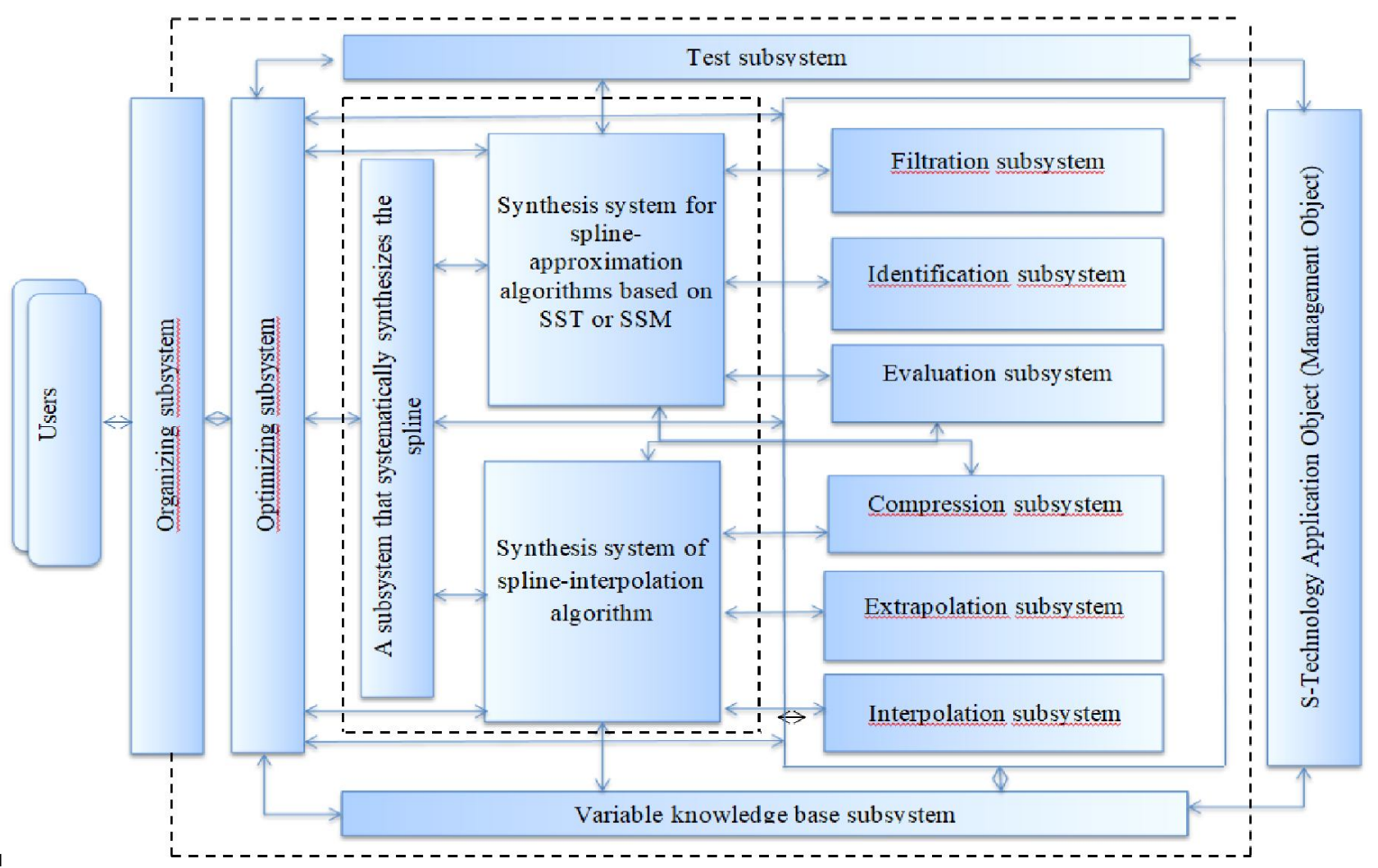

Figure 6: General classification of the proposed S-technology

Algorithms for the application of calculated splines with a given systematic content are created to solve the corresponding problems in the systems of interpolation, extrapolation, compression, evaluation, filtering and identification, which are functional modules.

The theory of spline serves as the logical basis of all the above subsystems and spline technologies. Each subsystem offers a defining, creative, and instrumental type. Each subsystem includes model process operations of information, material, and naturally combined information material.

\section{CONCLUSION}

The issue of interpolation plays an important role in the analysis of digital signal processing. In this article, a spline model is constructed and the interpolation process is performed in the analysis of signals measured from technological processes. The results showed that the spline function method had a high accuracy in interpolating signals (Figure 2). The basic spline model has the following features:

- good proximity to the object when interpolating signals;

- the construction of the model is much simpler than the classical polynomials;
- showed that the development of an algorithm for determining the parameters of the spline is simple and convenient.

Thus, it makes sense to use a variety of private support systems in conjunction with special functional subsystems that make up not only the general supply subsystems (with basic hardware and software) but also the specific functional support subsystems when creating the technology. The use of cubic spline models built for unequal intervals in digital signal processing gives good results.

\section{REFERENCES}

1. Samarskiy A.A., Gulin A.V.Chislennye metody, Moscow «Science», the main edition of Physico-mathematical literature, 1989.

2. Baxvalov N.S., Jidkov N.P., Kobelkov G.M. Chislennye method. - M .: Science, 1987.

3. Zavyalov Yu.S., Kvasov B.I., Miroshnichenko V.L., Method spline-function. Moscow .: Science, 1980. 352 p.

4. Mirzaev A.E. Improving the efficiency of digital signal processing algorithms based on spline functions. A dissertation for the degree of Doctor of Philosophy (PhD) in Technical Sciences. Toshkent 2019 y. 
5. Zaynidinov X.N., Bakhromov S.A., Azimov B.R. Construction of interpolated cubic spline model of biomedical signals. Generations of Muhammad al-Khwarizmi, № 4 (10), December 2019, pp. 14-17.

6. R. Steven Turley. Cubic Interpolation with Irregularly Spaced Points. Brigham Young University BYU ScholarsArchive. August 23, 2018.

7. Djananjay Singh., Madhusudan Singh., Hakimjon Zaynidinov. "Signal Processing Applications Using Multidimensional Polynomial Splines," Springer Briefs in Applied Sciences and Technology Series, Springer, Singapore, ISBN-978-981-13-2238-9. 2019.

8. Shumilov A.V. Analysis of the development and development of new software systems for the processing and interpretation of information on geophysical exploration wells. Vestnik PNIPU Geology. 2019. T.19.№2. S.162-174. DOI: 10.15593 / 2224-9923 / 2019.2.6.

9. Zaynidinov, H., Zaynutdinova, M., Nazirova, E. "Digital processing of two-dimensional signals in the basis of Haar wavelets" 2018 ACM International Conference Proceeding Series c. 130-133

https://doi.org/10.1145/3274005.3274023

10. Djananjay Singh, Madhusudan Singh, Hakimjon Zaynidinov "Signal Processing Applications Using Multidimensional Polynomial Splines", Springer Briefs in Applied Sciences and Technology Series, Springer, Singapore. ISBN-978-981-13-2238-9. 2019.

11. Zaynidinov H.N.,Mallayev O.U., Yusupov I "Cubic basic splines and parallel algorithms" 2020 International Journal of Advanced Trends in Computer Science and Engineering 9(3) c 3957-3960.

https://doi.org/10.30534/ijatcse/2020/219932020

12. Karimov, M., Tashev, K., Yoriqulov, M. "Problems of increasing efficiency of NIDS by using implementing methods packet classifications on FPGA" 2019 International Conference on Information Science and Communications Technologies: Applications, Trends and Opportunities, ICISCT 2019

https://doi.org/10.1109/ICISCT47635.2019.9011925

13. Zaynidinov, H., Juraev, J., Juraev, U. "Digital image processing with two-dimensional haar wavelets" 2020 International Journal of Advanced Trends in Computer Science and Engineering 9(3), c. 2729-2734

https://doi.org/10.30534/ijatcse/2020/38932020 